\title{
Comparative Performance of Single Layer and Multilayer Microwave Filters including the Influence of the Fabrication Process
}

\author{
Wesam Ali, Chunwei Min, and Charles Free \\ Advanced Technology Institute, University of Surrey \\ Guildford Surrey GU2 7XH, UK \\ W.Ali@surrey.ac.uk
}

\begin{abstract}
Modern fabrication processes offer the microwave circuit designer a wide range of techniques and materials for realizing passive planar components, such as bandpass filters. This paper investigates the effects that the fabrication process has on the performance of microwave planar circuits, so as to provide the circuit designer with useful practical information leading to the optimum choice of materials and processes. In order to compare the technologies, single-layer and multi-layer band-pass filter circuits were fabricated and tested at $25 \mathrm{GHz}$ and $2.5 \mathrm{GHz}$, respectively.
\end{abstract}

\section{Introduction}

Edge-coupled planar band-pass filters were chosen for this study because they are particularly sensitive to errors in the fabrication process [1]. Notably, in single-layer designs, errors in the gap size between the end sections of the filter have a significant effect on the electrical performance of the filter. The problem associated with fabricating small coupling gaps in a single layer circuit can be overcome by using a multi-layer structure, where the coupling is between overlapping conductors separated by dielectric. In a filter, this produces strong coupling between the elements of the filter, without the need for small gaps [2]. To some extent, the problem of fabricating small gaps has been exchanged for that of achieving high lateral resolution between the layers. However, with modern mask aligners it is relatively straightforward to achieve the required degree of resolution.

The two circuit technologies, namely polymer and photoimageable thick film chosen for the present study enable both single and multi-layer circuits to be fabricated. The photoimageable thick-film process enables the layers to be built up successively using a print-and-fire process. The polymer approach enables the circuit layers to be processed in parallel and finally stacked and laminated under relatively low temperature and pressure. In many ways this polymer technology is a competitor to the rather better established LTCC (low temperature co-fired ceramic) process, and offers potential advantages in terms of low-cost production.

Included in the paper is a design strategy for multilayer, edge-coupled, bandpass filters (ECBPFs). In single-layer structures, such as microstrip and stripline, ECBPFs are relatively easy to implement with working bandwidths up to $15-20 \%$. Wider bandwidths require tighter coupling between conductors, and this is where multilayer structures offer significant benefits.
Measurement data for an ECBPF multilayer design are presented, to validate the proposed design methodology.

\section{Design of ECBPF on Polymer Substrate}

Polymer substrates are normally supplied from the manufacturer pre-coated with thin copper conductor, which can be etched to form the required patterns using a conventional, high-quality $\mathrm{PCB}$ process.

In this study, a 4-section bandpass filter was designed with a centre frequency of $25 \mathrm{GHz}$ with a fractional bandwidth of $10 \%$, and a roll-off specified by $\left|S_{21}\right|^{2}<$ $30 \mathrm{~dB}$ at $20 \mathrm{GHz}$. The filter was fabricated on polymer substrate $\left(\varepsilon_{r}=2.5\right)$ having a thickness of $0.128 \mathrm{~mm}$. The physical dimensions of each of the coupled sections are shown in Table I.

TABLE I

PHYSICAL DIMENSIONS OF THE FILTER ON POLYMER SUBSTRATE AT $25 \mathrm{GHz}$

\begin{tabular}{|c|c|c|c|c|}
\hline Section & 1 & 2 & 3 & 4 \\
\hline Width (W) & 0.205 & 0.314 & 0.314 & 0.205 \\
\hline Space (S) & 0.018 & 0.071 & 0.071 & 0.018 \\
\hline Length (L) & 2.083 & 2.031 & 2.031 & 2.083 \\
\hline
\end{tabular}

(in $\mathrm{mm}$ )

After fabrication the filter was characterized using an HP 8510 vector network analyzer, with the filter mounted in a Wiltron Universal Test Fixture. Fig. 1 shows the simulated and measured responses of the designed filter. The simulated filter had a centre frequency of $25.065 \mathrm{GHz}$ with a $3 \mathrm{~dB}$ bandwidth of $3.97 \mathrm{GHz}(15.8 \%)$, and a roll-off of $\left|S_{21}\right|^{2}<-30 \mathrm{~dB}$ at $20 \mathrm{GHz}$. The maximum insertion loss in the passband is around $1.77 \mathrm{~dB}$. The measured circuit had a centre frequency of $25.45 \mathrm{GHz}$ with a $3 \mathrm{~dB}$ bandwidth of $3.65 \mathrm{GHz}(14.34 \%)$, and a roll off of $\left|S_{21}\right|^{2}<-30 \mathrm{~dB}$ at $21.8 \mathrm{GHz}$. Thus it is seen that the centre frequency of the fabricated filter has shifted by $1.53 \%$, with the bandwidth $1.46 \%$ less than the desired value.

To provide some traceability of the errors due to the fabrication process, mask and circuit dimensions were measured at various stages in the process. Table II shows the physical dimensions of the design, the dimensions on the mask and the dimensions of the filter after the fabrication. It is clear from Table II that the physical dimensions of the design almost match with the dimensions on the mask while there is a difference between the designed values and the fabricated circuit dimensions. The width and the length of each filter 
section decreased after the etching process while the spacings between each filter section became larger.

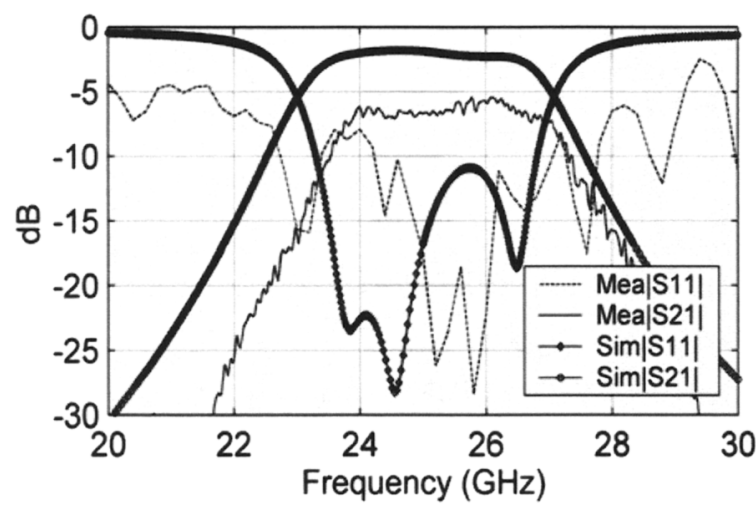

Fig. 1. Simulated and measured responses of the filter design on polymer at $25 \mathrm{GHz}$.

TABLE II

PHYSICAL DIMENSIONS OF THE FILTER ON POLYMER AT $25 \mathrm{GHz}$

\begin{tabular}{|c|c|c|c|c|}
\hline Section & 1 & 2 & 3 & 4 \\
\hline W - Design & 0.205 & 0.314 & 0.314 & 0.205 \\
\hline W - Mask & 0.205 & 0.214 & 0.214 & 0.205 \\
\hline W - Fabricated & 0.160 & 0.262 & 0.262 & 0.160 \\
\hline S - Design & 0.018 & 0.071 & 0.071 & 0.018 \\
\hline S - Mask & 0.017 & 0.0 .07 & 0.070 & 0.017 \\
\hline S - Fabricated & 0.054 & 0.121 & 0.121 & 0.054 \\
\hline L - Design & 2.083 & 2.031 & 2.031 & 2.083 \\
\hline L - Mask & 2.080 & 2.006 & 2.006 & 2.080 \\
\hline L - Fabricated & 2.072 & 1.928 & 1.928 & 2.072 \\
\hline
\end{tabular}

(in $\mathrm{mm}$ )

Fig. 2 illustrates the effect of undercutting in the fabrication process, and clearly this is going to be a significant issue in realizing filters with high, predictable performance.

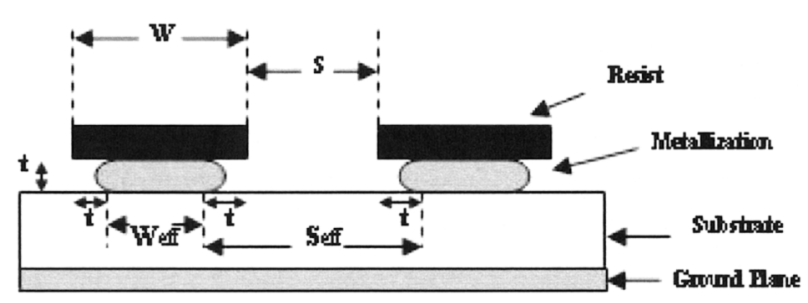

Fig. 2. The effective dimensions after the etching process.

If we assume an etching ratio of unity, the following design equations in (1) may be used to compensate for the phenomenon of undercutting by increasing the width $(W)$ of the conductors on the photo-mask

$$
W=W_{d}+2 t \quad S=S_{d}-2 t \quad L=L_{d}+2 t
$$

where $W_{d}, S_{d}, L_{d}$, are the design dimensions, and $t$ is the thickness of the metal (copper) used in the circuit. The measured thickness of the copper was $0.017 \mathrm{~mm}$. The effect of undercutting of the metallization was probably the main reason for the reduction of the measured bandwidth, and for the poor return loss. The latter quantity is very dependent on the conductor widths and spacings being correct so as to maintain the correct odd and even mode impedances. The losses due to the dielectric material and bulk conductors had been included in the simulation, although there may be additional insertion loss due to the surface roughness of the final circuits.

\section{Design of ECBPF on Alumina Substrate}

For this design, an edge-coupled band-pass filter in single layer format was fabricated on typical $96 \%$ alumina substrate having a thickness of $0.254 \mathrm{~mm}$ and a relative permittivity $\left(\varepsilon_{r}\right)$ of 9.8 . A photoimageable thickfilm (PTF) process was used to realize the conductor pattern. The filter had been designed with a centre frequency of $25 \mathrm{GHz}$ with a required fractional bandwidth of $10 \%$, and a roll-off specified by $|\mathrm{S} 21|<-30 \mathrm{~dB}$ at $20 \mathrm{GHz}$. The physical dimensions of each of the coupled sections are shown in Table III.

\section{TABLE III}

PHYSICAL DIMENSIONS OF THE FILTER ON ALUMINA SUBSTRATE AT $25 \mathrm{GHz}$

\begin{tabular}{|c|c|c|c|c|}
\hline Section & 1 & 2 & 3 & 4 \\
\hline Width (W) & 0.127 & 0.210 & 0.210 & 0.127 \\
\hline Space (S) & 0.066 & 0.203 & 0.203 & 0.066 \\
\hline Length (L) & 1.130 & 1.078 & 1.078 & 1.130 \\
\hline
\end{tabular}

$\mathrm{mm})$

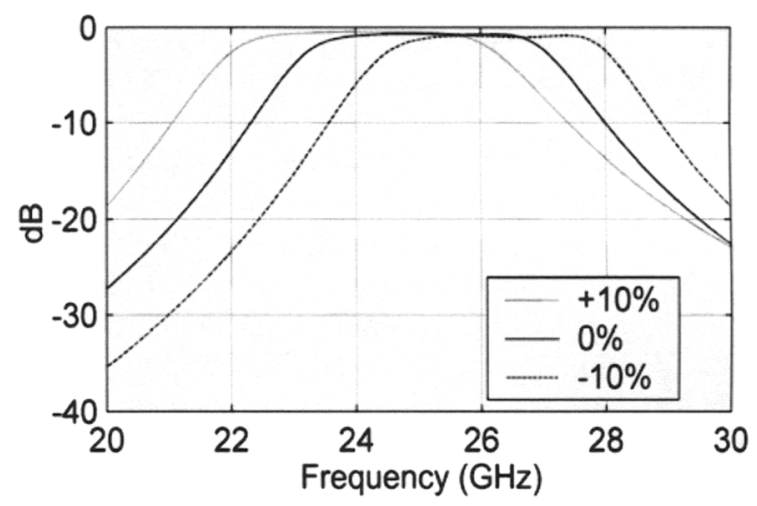

Fig. 3. Simulation of filter insertion loss showing effects of various percentage errors in the conductor widths and spacings.

Due to the variations in circuit geometries that can occur during thick film processing, analysis of the effects on the fabrication errors is essential. Simulation data shown in Fig. 3 and Fig. 4 indicate how the insertion loss and matching of the filter vary with $\pm 10 \%$ change in circuit dimensions. A $10 \%$ decrease of the length and width of the filter elements results in a narrowing of the bandwidth of about $15.12 \%$ with worse roll-off, and a slight downward shift of the centre frequency of about 
$4.41 \%$. A $10 \%$ of increase of dimensions results in a wider bandwidth of about $18.28 \%$ with better roll-off, and slightly upward shift of the centre frequency of around $4 \%$. This analysis gives a useful insight into the effect of shrinkage during the firing process on the filter's electrical performance, and clearly shows the need for the effects of the fabrication process to be considered at the design stage.

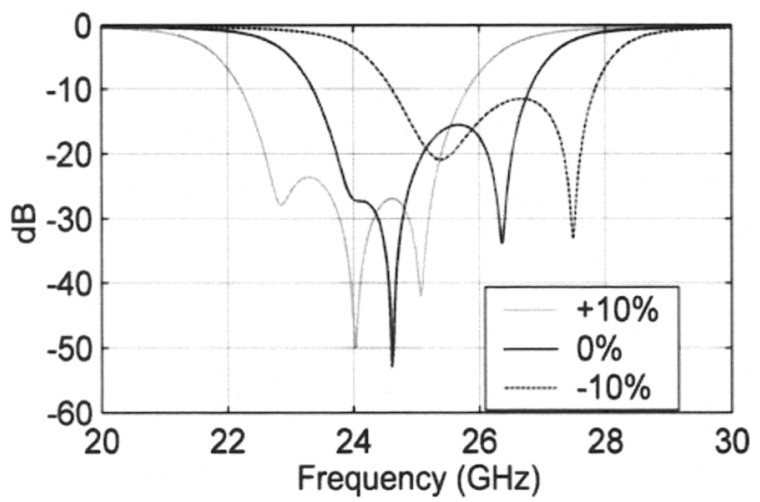

Fig. 4. Simulated return loss results for different fabrication errors.

Fig. 5 shows a comparison between the simulated and measured responses of the filter. The filter had a centre frequency of $25.135 \mathrm{GHz}$ with the $3 \mathrm{~dB}$ bandwidth about $3.99 \mathrm{GHz}(15.87 \%)$ and the roll-off of $\left.|| S_{21}\right|^{2}<-27 \mathrm{~dB}$ at $20 \mathrm{GHz}$. The maximum insertion loss in the passband is around $0.031 \mathrm{~dB}$. The return loss is below $15 \mathrm{~dB}$ which represents good matching of the circuit around the operating frequency band. The measured circuit had a centre frequency of $26.75 \mathrm{GHz}$ with $3 \mathrm{~dB}$ bandwidth which to be about $3.5 \mathrm{GHz}(13.08 \%)$ and roll off of $\left|S_{21}\right|^{2} \mid<-30 \mathrm{~dB}$ at $22 \mathrm{GHz}$ with insertion loss of $6.06 \mathrm{~dB}$. The return loss is below $10 \mathrm{~dB}$ which represents reasonably good matching of the circuit around the operating frequency band and meets the required specification. It is seen from the results that the centre frequency shifts by $1.11 \%$ and the bandwidth reduces slightly by $2.79 \%$.

It is seen from the results that the bandwidth reduces slightly and this was attributed to shrinkage of the conductors during firing, which resulted in an increase in the spacing of the conductors in each section of the filter. The slight shift in the centre frequency may have been due to shrinkage effects, but more likely to incorrect compensation for electromagnetic fringing at the end of each section. Although the losses due to the dielectric material and bulk conductors had been included in the simulation, the additional insertion loss may be due to the surface roughness of the fired circuits. It was observed that the insertion loss was higher for the thick-film filters, compared to those made on polymer, where the copper conductor was laminated onto the polymer. In these circumstances the thick-film surface would be expected to be somewhat rougher than that of the copper. This agrees with results published elsewhere [3] that show that the conductor loss at these frequencies is greater than that in the dielectric. This effect becomes increasingly significant at mm-wave frequencies.

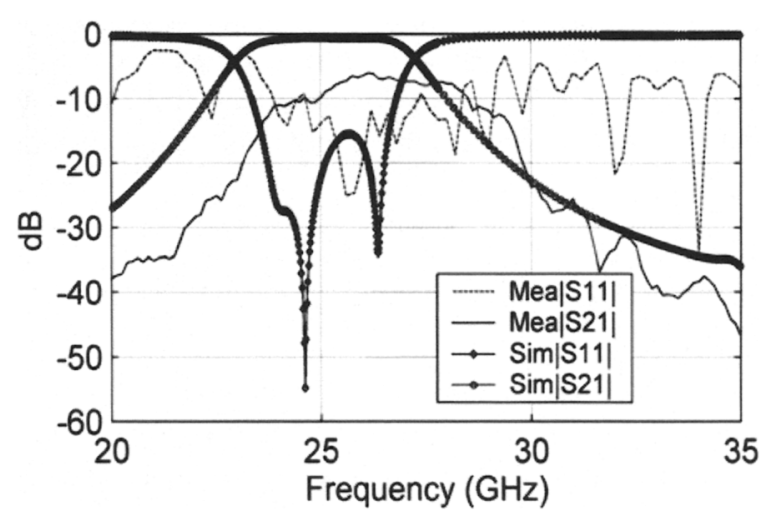

Fig. 5. Simulated and measured responses of the filter design on alumina at $25 \mathrm{GHz}$.

\section{Multilayer ECBPF Design}

Fig. 6 shows configuration of the proposed multilayer microstrip coupled-line filter. The coupling was achieved between asymmetrical conductors on different layers, with the configuration shown in Fig. 7.

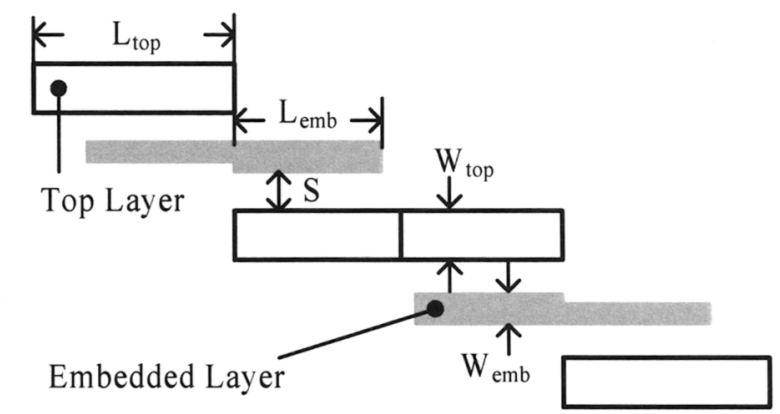

Fig. 6. Configuration of the proposed multilayer microstrip coupled-line filter.

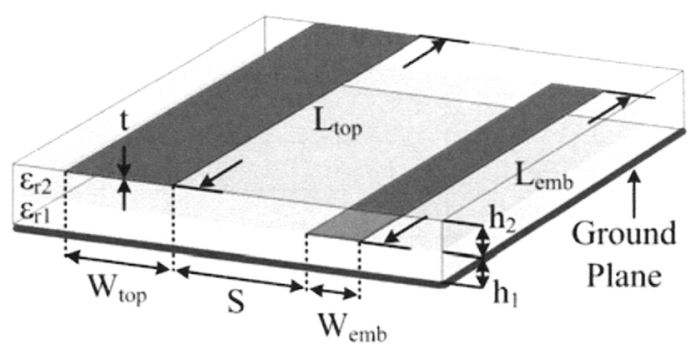

Fig. 7. Asymmetric microstrip coupled-line structure.

When the coupled lines are positioned on different dielectric layers they become physically asymmetric because of the need to maintain identical line impedances, with the same propagation characteristics. Hence these lines no longer support pure even and odd modes. Rather, the two excitation modes become dependent, corresponding to the $c$ - and $\pi$-modes. A full-wave based analysis in conjunction with the specified modal 
parameters enabled a procedure for the filter design to be developed. There are three main electrical parameters used for the design of coupled-line structures, namely, modal impedance, effective dielectric constant, and mutual coupling coefficient. Modal impedances $\left(Z_{c, \pi}\right)$ decide the corresponding widths for the top and embedded lines, which can be obtained using equation (2).

$$
Z_{0}=\sqrt{Z_{c t} Z_{\pi t}}=\sqrt{Z_{c e} Z_{\pi e}}
$$

The electrical length $(\theta)$ of the coupled lines is theoretically a quarter guide-wavelength $\left(\lambda_{g} / 4, \pi / 2\right)$ long. Open-end effects of this structure can be represented by an additional length $(\Delta L)$ of the line that needs to be subtracted from the nominal $\lambda_{\mathrm{g}} / 2$ length. The physical lengths $(L)$ of the lines used in the filter design are thus obtained by calculating the effective dielectric constant $\left(\varepsilon_{e f f}(f)\right)$ at the frequency of interest through the following equation

$$
L=\left(\frac{c}{2 \pi f}\right)\left(\frac{\theta}{\sqrt{\varepsilon_{\text {eff }}(f)}}\right)-\Delta L
$$

where $c$ is the speed of light.

The coupling factor $\left(k_{c}\right)$ may be obtained according to the modal impedances appropriately chosen for the two lines using

$$
k_{c(d B)}=20 \log \left(\frac{Z_{c t, e}-Z_{\pi t, e}}{Z_{c t, e}+Z_{\pi t, e}}\right)
$$

TABLE IV

DIMENSIONS OF THE DESIGN AT $2.5 \mathrm{GHz}$

\begin{tabular}{|c|c|c|c|c|}
\hline Sec., $n$ & 1 & 2 & 3 & 4 \\
\hline$W_{\text {top }}$ & 2.610 & 3.110 & 3.110 & 2.610 \\
\hline$L_{\text {top }}$ & 20.950 & 20.850 & 20.850 & 20.950 \\
\hline$W_{e m b}$ & 1.180 & 1.430 & 1.430 & 1.180 \\
\hline$L_{e m b}$ & 19.800 & 19.830 & 19.830 & 19.800 \\
\hline$S$ & -0.700 & 0.140 & 0.140 & -0.700 \\
\hline
\end{tabular}

(in $\mathrm{mm}$ )

The proposed filter designs were realized using RT/duroid ${ }^{\circledR} 5880$ material with $\varepsilon_{r 1}=\varepsilon_{r 2}=2.2$ and $h_{l}=h_{2}=0.508 \mathrm{~mm}$. The design frequency was $2.5 \mathrm{GHz}$. Table IV summarizes the physical dimensions of the filters at $2.5 \mathrm{GHz}$ and Fig. 8 shows the simulated and measured responses. The measured bandwidth and insertion loss of the design were found to be $20.4 \%$ and $0.6 \mathrm{~dB}$, which was in close agreement with the simulated result. The results also emphasized the wide bandwidths possible with the multilayer configuration. The mismatch within the passband may be due to errors in the fabrication process, including layer-to-layer registration errors. These effects become pronounced when the operating frequency of the filter is higher, extending into the mm-wave band.

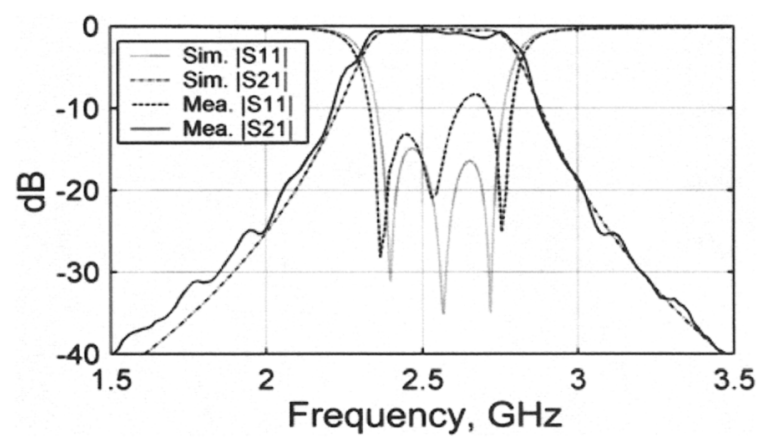

Fig. 8. Responses of the filter design at $2.5 \mathrm{GHz}$.

\section{Conclusions}

The work has identified those aspects of the fabrication process that have most influence on the performance of a microwave circuit. Furthermore, the work has indicated that selecting substrate and conductor materials consideration must be given to the limitations of the associated fabrication process.

A design procedure for edge-coupled multi-layer filters has been developed, and validated through practical measurement. The results for the multi-layer filter have reinforced the benefits of this type of circuit structure, particularly in overcoming the limitations of conventional, single-layer components. Using the multilayer formats, high quality components with wide bandwidths ( $>20 \%$ in our case) are possible with lowcost fabrication processes.

\section{References}

1. Edwards, T. C., and Steer, M. B., Foundations of Interconnect and Microstrip Design, J. Wiley \& Sons, $3^{\text {rd }}$ ed., (2000).

2. Tsai, C.-M., and Gupta, K. C., "A Generelized Model for Coupled Lines and Its Applications to Two-Layer Planar Circuits," IEEE Tranactions on Microwave Theory \& Technique, Vol. 40, No. 12 (1992), pp. 2190-2199.

3. Henry, M., and Free, C., "Electrical Charecterization of LTCC Coplanar Lines up to $110 \mathrm{GHz}$," European Microwave Conf, Manchester, UK, 2006.

4. Pozar, D. M., Microwave Engineering, J. Wiley \& Sons, $3^{\text {rd }}$ ed., (2005).

5. Gupta, K. C., Garg, R., Bahl, I., and Bhartia, P., Microstrip Lines and Slotlines, Artech House, (1996). 